(C) 1996 IEEE. Personal use of this material is permitted. However, permission to reprint/republish this material

for advertising or promotional purposes or for creating new collective works for resale or redistribution to servers

or lists, or to reuse any copyrighted component of this work in other works must be obtained from the IEEE.

\title{
TRANSVERSE-LONGITUDINAL ENERGY EQUILIBRATION IN A LONG UNIFORM BEAM"
}

\author{
I. Haber, Naval Research Laboratory, Washington, DC 20375 USA \\ D. A. Callahan, A. Friedman, D. P. Grote, A. B. Langdon \\ Lawrence Livermore National Laboratory, Livermore, CA 94550 USA
}

\section{Abstract}

Evidence is presented to show the transfer, via a collective electrostatic instability, of transverse energy to the longitudinal direction if the axial temperature of a beam is sufficiently low. Two-dimensional r,z electrostatic PIC simulations of a long coasting space-charge-dominated beam propagating in a uniform focused channel are presented to illustrate, for typical parameters, the development of a coherent mode structure with an axial wavelength comparable to the beam diameter, and growth times comparable to the betatron period.

\section{INTRODUCTION}

In many accelerator systems it is important to carefully control the phase space volume occupied by the beam. For example, in a heavy ion accelerator which is to be used for the ignition of a thermonuclear pellet, both the transverse beam emittance and the spread in longitudinal velocities are constrained by the requirement for focusing sufficient power density onto the target. While the constraints on the transverse and longitudinal phase space are generally related in a complex way by the details of the accelerator design, it is important to limit the growth of both.

If it is noted that the addition of equal energy to each beam particle during acceleration results in a decrease in the longitudinal velocity spread in the beam frame, while the same reduction does not occur in the transverse direction, it is easy to envision the development of a substantial anisotropy in the beam temperature. While the very low beam collision frequency precludes any thermodynamic equipartitioning of the temperature anisotropy on a time scale comparable to the beam lifetime, a collective mechanism that can transfer energy from the transverse to longitudinal directions is described here.

Previous examination $[1,2]$ of the behavior of beam bunches, where the length of the bunch is comparable to its radius, has identified possible collective mechanisms which can transfer energy between the transverse and longitudinal direction so as to reduce a temperature anisotropy. More recently, three-dimensional simulation studies $[3,4]$ of spacecharge-dominated beam dynamics have also shown evidence of a collective mechanism for energy transfer, even when the bunch length is much longer than the beam diameter. These simulations suggest that the collective mechanism does not depend on the bunch length being comparable to beam diameter. The work discussed here attempts to clarify the nature of that mechanism by examining a space-chargedominated beam with a transverse temperature greater than the longitudinal temperature, but in the simpler case of axisymmetric geometry and a uniform focusing channel.

As will be discussed below, the simulations show that an instability develops which transfers energy from the transverse to the longitudinal direction. The unstable mode in the example shown appears to behave like a localized envelope oscillation, with a length comparable to the beam diameter, that is driven unstable by the transverse energy. This mode appears to be only one of the potentially unstable, and possibly coupled, modes of the anisotropic beam system.

\section{AXISYMMETRIC SIMULATIONS}

For simplicity, the investigation described here is restricted to an axisymmetric space-charge-dominated beam in a focusing system which is linear and uniform in the longitudinal direction. The beam self-fields are assumed to be electrostatic. For further simplification, a relatively small, in the examples here $0.32 \mathrm{~m}$, section of the beam is examined using periodic boundary conditions. Figure 1 is a plot of the $z$ (longitudinal) rms. velocity of the beam particles as a function of the distance propagated by the beam as it is transported.

The beam shown in Fig. 1 is a 4.8 A beam consisting of singly charged ions with mass of 12 AMU and energy of 10 $\mathrm{MeV}$. A linear inward focusing force, independent of longitudinal position, is employed, corresponding to a $7.2 \mathrm{~m}$ low-current betatron wavelength, which maintains a matched beam radius of $0.023 \mathrm{~m}$, in the $0.05 \mathrm{~m}$ radius conducting pipe. The beam particles have been given an initially semi-Gaussian (uniform in space Gaussian in velocity) distribution with a transverse emittance of $2.2 \times 10^{-4} \mathrm{~m} \cdot \mathrm{rad}$. The initial conditions represent sufficient space charge to depress the transverse phase advance by a factor of 2.35 were the beam initially a $\mathrm{K}-\mathrm{V}$ distribution with the same rms. quantities. The initial thermal velocity in the longitudinal direction is $10^{3} \mathrm{~m} / \mathrm{sec}$.

The numerical simulations were performed using the electrostatic r,z WARP [5] particle-in-cell simulation code. The particular simulation shown in Fig. 1 employed $224 \mathrm{~K}$ macroparticles. The grid consisted of 32 radial and 512 axial zones, with Gaussian smoothing employed in the axial direction to suppress the longitudinal grid instability

\footnotetext{
* Work performed under the auspices of the United States Department of Energy by NRL and LLNL under contracts

DE-AI02-93ER40799, DE-AI02-94ER54232, and W-7405-ENG-48.
} 
sometimes observed in the simulation of longitudinally cold beams.

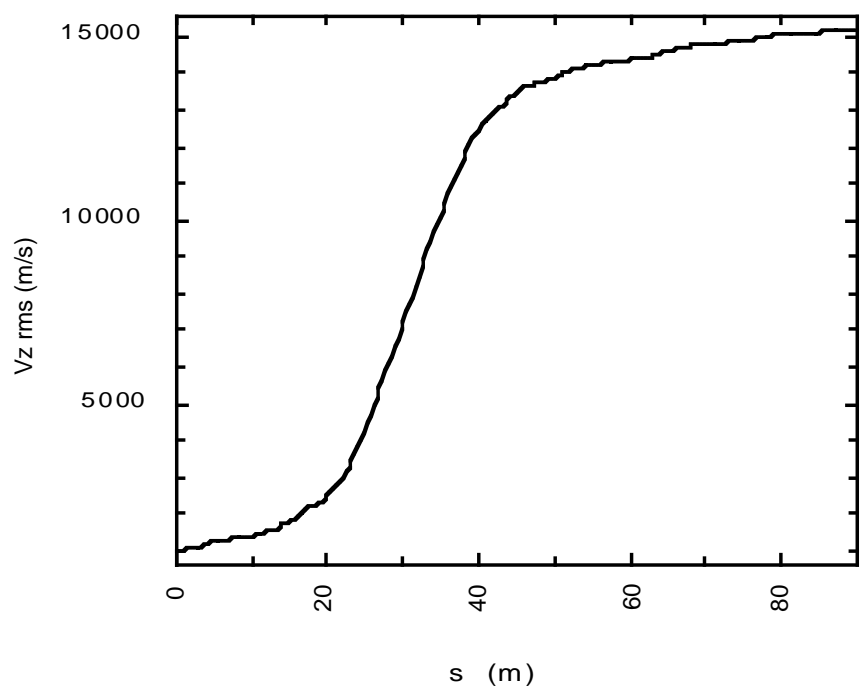

Fig 1. Longitudinal rms. velocity plotted against the distance propagated by the beam. Energy from the initially semi-Gaussian transverse distribution is transferred to the longitudinal direction.

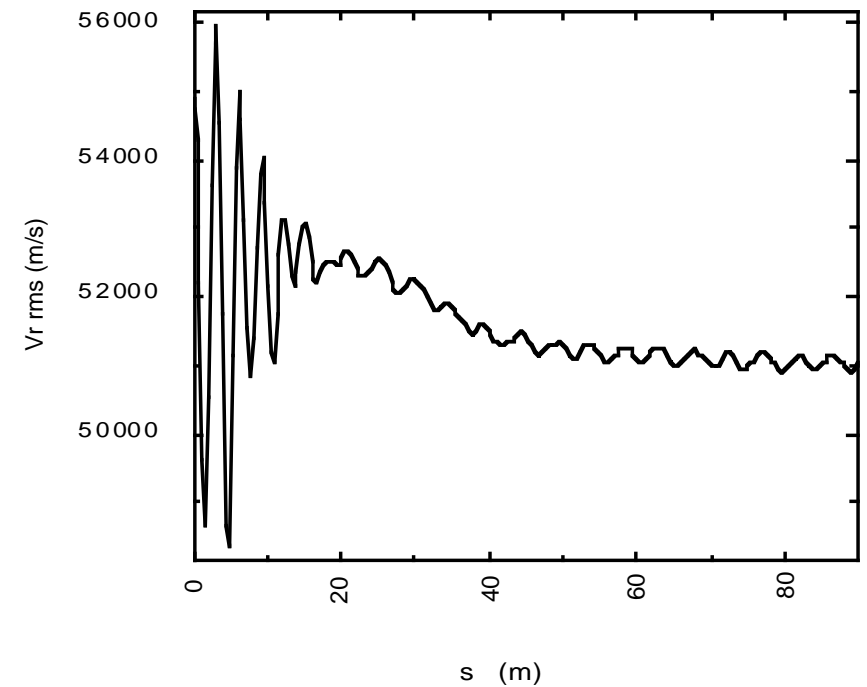

Fig. 2. Evolution of the transverse rms. velocity, showing the initial fluctuations that result from a lack of detailed force balance when the beam is prepared with an initially semi-Gaussian distribution.

The growth in longitudinal thermal energy shown in Fig. 1 can be separated into three phases, consisting of an initial short period of slow growth followed by a period of somewhat more rapid growth, and finally a slowing of this rapid growth. Because it is conjectured that the mechanism for transfer of energy is a result of a collective instability, the initial growth phase is problematic, since an instability which is not explicitly seeded will tend to grow from some small initial perturbation which is due to a nonuniformity in the initial distribution. A possible explanation for this initial behavior can be found from the examination of the transverse dynamics, as is illustrated by the plot in Fig. 2 of the transverse rms. velocity as the beam propagates down the transport line.

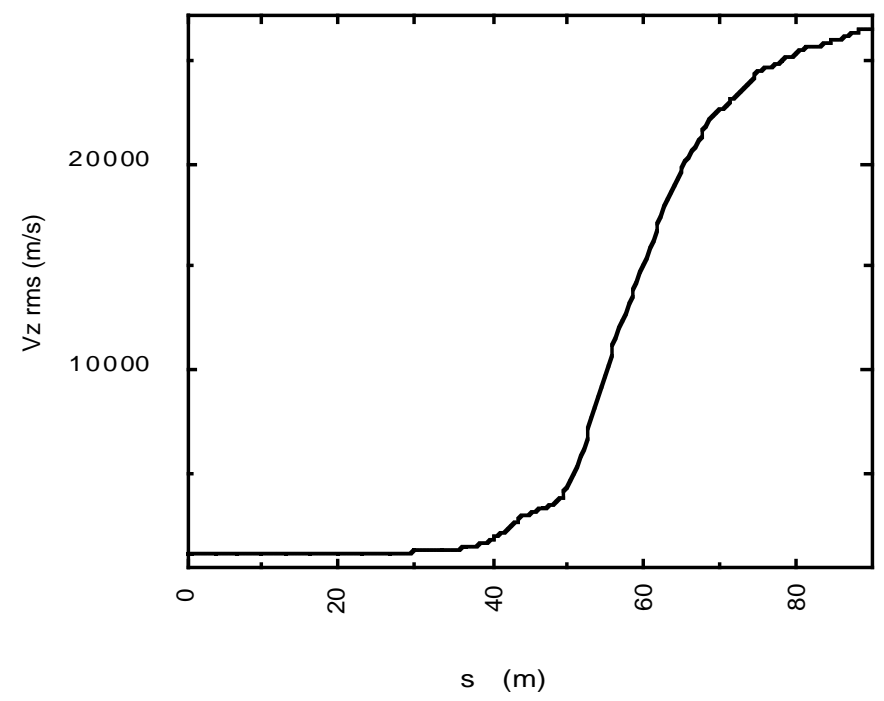

Fig 3. Longitudinal rms. velocity plotted against the distance propagated, when the beam is given an initially $\mathrm{K}$ $\mathrm{V}$ distribution. Note the delayed onset of growth in the longitudinal thermal energy.

While a beam with an initially semi-Gaussian transverse distribution can be close to a steady state distribution, especially in the limit of small emittance, this distribution is not in local force balance with the external focusing force. An initial transient therefore will occur until the beam relaxes to an equilibrium. Since the beam in the simulation is represented by a number of particles which is far less than the number in an actual beam, the resulting granularity in the distribution causes numerical collisions which can couplemomentum from the internal dynamics of the transverse beam redistribution to the longitudinal direction and therefore heat the beam longitudinally. A numerical cause for the initial growth in longitudinal energy is supported by the fact that the growth rate does decrease with an increase in the number of simulation particles used to represent the beam.

In order to remove the complications associated with any initial redistribution of a beam not in equilibrium, simulations were performed employing an initially K-V distribution, for which the beam is in local force balance with the focusing forces. Figure 3 is a plot of the evolution of the rms. $z$ velocity for an initially $\mathrm{K}-\mathrm{V}$ beam with the same rms. parameters as in the previous case shown in Figs. 1 and 2 . Absent the redistribution to equilibrium present in the initially semi-Gaussian beam, the longitudinal velocity shows the quiescent period and exponentiation which is characteristic of an unstable mode growing from the small initial fluctuations in the initial distribution function. The smoother initial behavior can also be noted by examining the rms. radial velocity component shown in Fig. 4. The largeinitial fluctuations are now absent and the transverse cooling, as the energy is transferred to the longitudinal direction, is now evident. 
A further interesting facet of the instability mechanism is the creation of large fluctuations in the beam current, as shown in Fig. 5, which is a plot of the current after the beam has propagated 60 meters, or 8.3 betatron periods. Note that the characteristic scale of these fluctuations is on the same order as the beam radius.

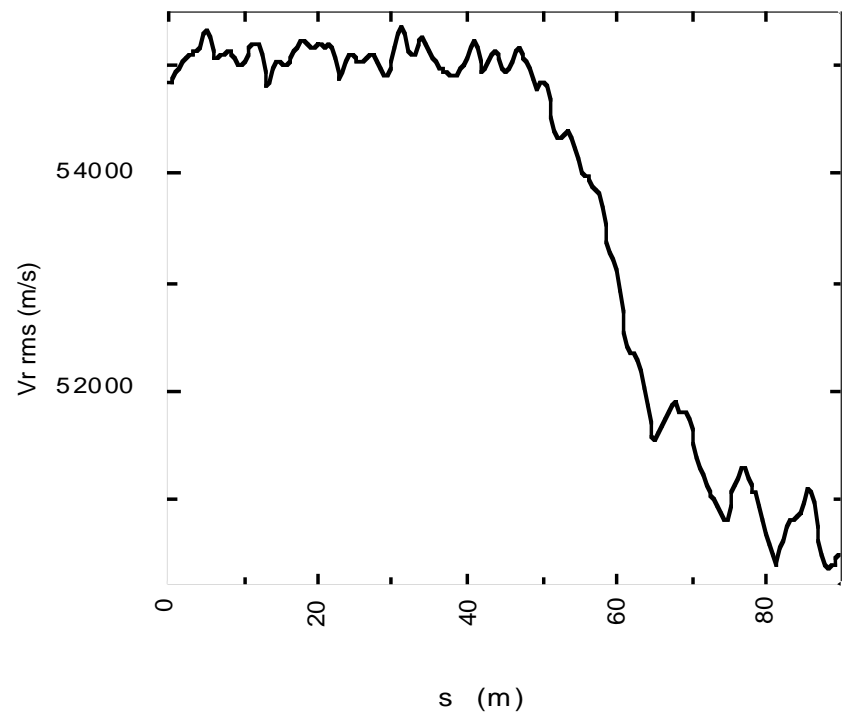

Fig. 4. Evolution of the transverse rms. velocity for the beam with an initially $\mathrm{K}-\mathrm{V}$ distribution, showing the smooth transfer of energy from the transverse to the longitudinal direction.

\section{DISCUSSION OF SIMULATIONS}

The two simulations discussed above are representative of several that have been conducted to examine details of an instability which is responsible for the transfer of energy from the transverse to longitudinal dimensions when there is a substantial anisotropy in beam temperature. Limited space however, restricts the discussion here to describing some of the more important features which were observed.

One significant feature seen in the linear phase of the instability is that the transverse dependence of the self-electric fields within the beam strongly resemble the $\mathrm{J}_{1}(\mathrm{kr})$ and $\mathrm{J}_{0}(\mathrm{kr})$ Bessel functions for $E_{r}$ and $E_{Z}$ respectively. For the parameter regime shown here, the value of $\mathrm{k}$ for the unstable mode is such that the longitudinal electric field, but not the transverse, reverses direction within the beam. Self-electric fields with this transverse dependence are associated with the solutions of Poisson's equation that have a real (decaying or growing) exponential dependence on $z$ rather than being propagating modes. The local nature of the growing modes that this implies was verified by seeding the instability by a local perturbation in the beam radius. Preliminary analysis of the characteristic frequencies of such an envelope perturbation, which is localized in the axial direction, show that oscillation frequencies are consistent with what is required for the fields to resonate with the particle betatron oscillations, including the space charge depression. The beam dynamics appear to be complicated, however, by a coupling to the longitudinal space charge waves that will propagate the energy in the growing modes. Though the mechanism appears to be similar to the Harris instability[6], with the betatron oscillations replacing the cyclotron motion in that case, the finite transverse geometry in the present case appears to substantially modify the dynamics.

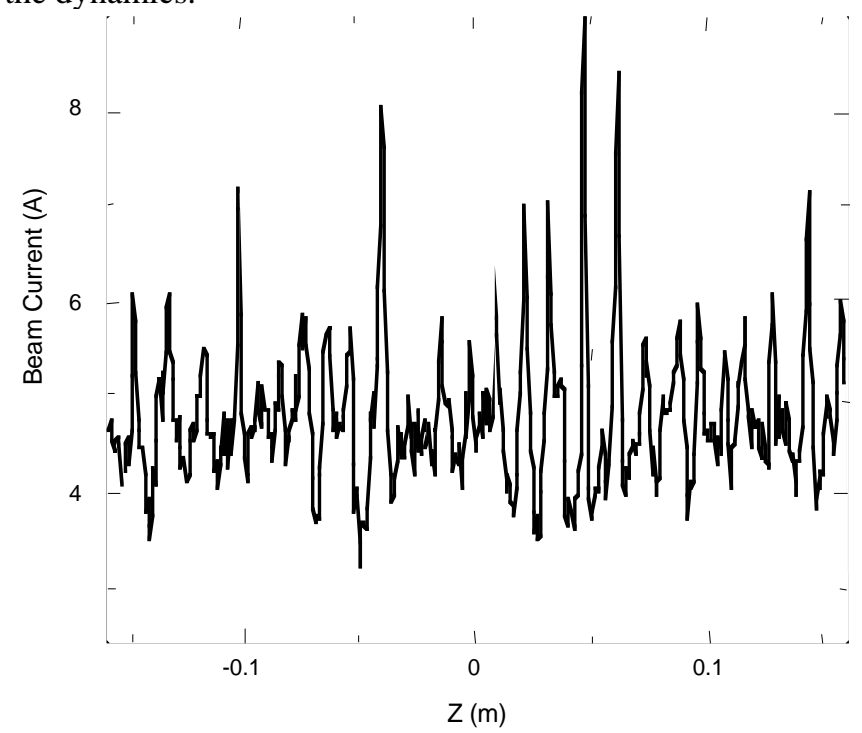

Fig. 5. Beam current plotted against axial position within the beam, after the beam has propagated $60 \mathrm{~m}$.

\section{REFERENCES}

[1] I. Hofmann, "Emittance Growth of Beams Close to the Space Charge Limit," IEEE Tran. Nucl. Sci. NS-28, 2399 (1981).

[2] R. A. Jameson, "Beam Intensity Limitations in Linear Accelerators," op. cit. 2408.

[3] Alex Friedman, David P. Grote, and Irving Haber, "ThreeDimensional Particle Simulation of Heavy-Ion Fusion Beams," Phys. Fluids B 4, 2203 (July, 1992).

[4] A. Friedman, D. A. Callahan, D. P. Grote, A. B. Langdon, and I. Haber, "Studies of Equilibration Processes in Heavy Ion Beams," Bull. Am. Phys. Soc., 35, 9, 2121 (Oct. 1990)

[5] Debra A. Callahan, A. Bruce Langdon, Alex Friedman, and Irving Haber, "Longitudinal Beam Dynamics for Heavy Ion Fusion," Proc. 1993 Particle Accel. Conf., 730 (IEEE, 1993).

[6] E. G. Harris, "Unstable Plasma Oscillations in a Magnetic Field," Phys. Rev. Letters, 2,34 (1959). 